\title{
Dimensions of poly(trimethylene oxide) chains in a theta-solvent
}

\author{
D. S. Chiu, Y. Takahashi* and J. E. Mark \\ Department of Chemistry and the Macromolecular Research Center, University of Michigan, Ann \\ Arbor, Michigan, 48109, USA \\ (Received 26 February 1976)
}

\begin{abstract}
Fractions of poly(trimethylene oxide) $\left.\mathrm{HCH}_{2}\right)_{3}-\mathrm{O}_{x}$ of relatively high degree of polymerization $x$ were studied in liquid-liquid phase equilibria in the poor solvent cyclohexane. The results thus obtained indicate that the ideal or theta-temperature for this system is $27.0^{\circ} \pm 0.5^{\circ} \mathrm{C}$. Intrinsic viscosities measured under these conditions were used to obtain a value for the characteristic ratio $\left\langle v^{2}\right\rangle_{0} /\left.n\right|^{2}$ of the unperturbed dimensions of these chains relative to the number of skeletal bonds and the average square of their length. The resulting value, $3.86 \pm 0.08$, is unusually low, a fact which may be attributed to the high degree of conformational randomness in this chain molecule. Consideration of poly(trimethylene oxide) to be an alternating copolymer of ethylene and oxymethylene provides some additional information of interest. It clearly demonstrates that a chemical copolymer can have configuration-dependent properties which are vastly different from those of its parent homopolymers.
\end{abstract}

\section{INTRODUCTION}

Poly(trimethylene oxide) $\left(\mathrm{CH}_{2}-\mathrm{CH}_{2}-\mathrm{CH}_{2}-\mathrm{O}\right)_{x}$ is one of the most interesting chain molecules which may be studied with regard to its spatial configurations in the random-coil state. The polymer may be prepared to relatively high degrees of polymerization $x^{1}$, and has a rather low melting point $\left(35^{\circ} \mathrm{C}\right)^{2}$; its characterization from the experimental point of view should therefore be straightforward. In addition, its investigation ${ }^{3-5}$ in terms of the rotational isomeric state theory ${ }^{4}$ of chain configurations is facilitated by two important features of this polyoxide chain. Its repeat unit has a very simple structure and most of the intramolecular interactions occurring in this molecule also occur in other members of the $\left(\left(\mathrm{CH}_{2}\right)_{y}-\mathrm{O}\right\}_{x}$ series, several of which have now been extensively studied with regard to their configuration-dependent properties ${ }^{4-6}$. Finally, preliminary evidence ${ }^{1}$ indicates that poly(trimethylene oxide) in the random-coil state is an unusually compact chain molecule, judging from approximate values of its unperturbed dimensions as estimated from viscosity measurements in a thermodynamically good solvent.

For the above reasons, the present study was undertaken to determine a reliable experimental value of the characteristic ratio $\left\langle r^{2}\right\rangle_{0} / n l^{2}$ of poly(trimethylene oxide), where $\left\langle r^{2}\right\rangle_{0}$ is the chain dimension as unperturbed by excluded volume effects ${ }^{1,7}, n$ is the number of skeletal bonds, and $l^{2}$ is the average square of their length. The approach taken, the study of the molecules in a theta $(\theta)$ solvent, where excluded volume interactions are known to be nullified $^{7}$, is probably the most reliable method presently available for this purpose. The temperature at which a suitably poor solvent acts as a $\theta$-solvent for poly(trimethylene oxide) will be located by measurements of the critical solution temperatures for liquid-liquid phase equilibria of solutions of the polymer, obtained as a function of its degree of polymerization ${ }^{7}$. Intrinsic viscosities measured in this solvent at the $\theta$-temperature thus established then

* Permanent address: Faculty of Science, Osaka University, Toyonaka, Osaka 560, Japan. directly yield values of the unperturbed dimensions and the characteristic ratio, without the need for approximate corrections for chain expansion due to excluded volume interactions.

Since there has been a considerable amount of interest in the configuration-dependent properties of chemical copolymers $^{8-12}$, it should be noted that poly(trimethylene oxide) may be thought of as an alternating copolymer of ethylene and oxymethylene. It is therefore obviously of considerable interest to compare its statistical properties with those of polyethylene ${ }^{4}$ and polyoxymethylene ${ }^{4,13,14}$

\section{EXPERIMENTAL}

The five fractions of poly(trimethylene oxide) employed in the present study were among those studied previously in a thermodynamically good solvent, and their preparation is thus described elsewhere ${ }^{1}$. Their number-average molecular weights $M_{n}$, which had been determined by osmometry in benzene at $30^{\circ} \mathrm{C}^{1}$, are listed in Table 1 .

A preliminary survey of solvents indicated that cyclohexane should serve as a $\theta$-solvent ${ }^{7}$ for poly(trimethylene oxide) in the vicinity of room temperature. Precipitation temperatures, $T_{p}$, for liquid-liquid separations in the reagent-grade solvent were therefore determined for the five fractions listed in Table 1, augmented by two others (F1 and $F 7^{1}$, having values of $M_{n} \times 10^{-3}, 53.5$ and 153.0 , res-

Table 1 Experimental results on poly(trimethylene oxide)

\begin{tabular}{lccc}
\hline Fractiona & $M_{n} \times 10^{-3 b}$ & $\begin{array}{l}{[\eta] c} \\
\langle\mathrm{~d} l / g)\end{array}$ & $\left\langle r^{2}\right\rangle_{0} /\left.n\right|^{2}$ \\
\hline F2 & 65.1 & 0.275 & 3.78 \\
F3 & 87.5 & 0.316 & 3.76 \\
F4 & 109.1 & 0.380 & 3.95 \\
F8 & 161.1 & 0.465 & 3.97 \\
F10 & 292.5 & 0.591 & 3.82 \\
& & & Average $3.86 \pm 0.08$ \\
\hline
\end{tabular}

a Detailed information on these fractions is given in ref 1. bobtained from osmotic pressure measurements in benzene at $30^{\circ} \mathrm{C}$; see ref 1 . $\mathrm{C}$ In the $\theta$-solvent, cyclohexane, at $27.0^{\circ} \mathrm{C}$; present results 


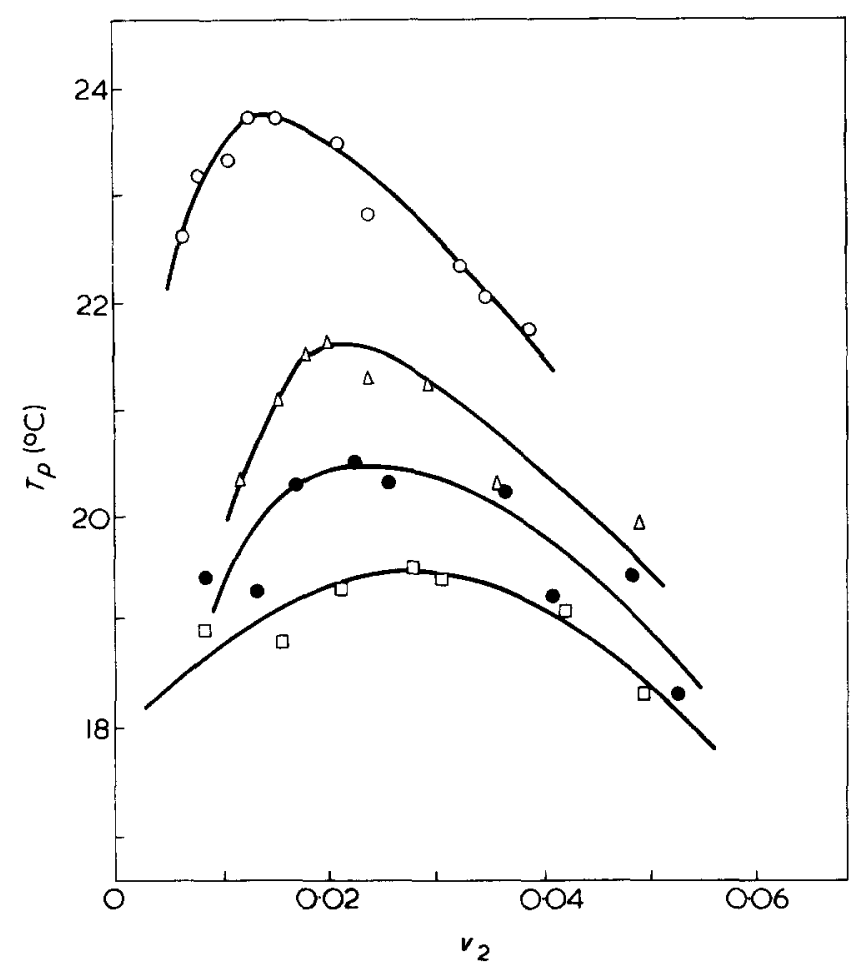

Figure 1 Some typical binodials characterizing liquid-liquid separations in the poly (trimethylene oxide)-cyclohexane system. The fractions represented are described in Table 1, and in a previous study ${ }^{1} .: 0, F_{10} \triangle F_{7} ;, F 4 ; \square, F 2$

pectively). Values of $T_{p}$ were determined as a function of composition, for all seven fractions, using simple visual inspection, in the usual manner ${ }^{15}$. The volume fraction $v_{2}$ of polymer in each solution at $23^{\circ} \mathrm{C}$ was obtained from the weights of polymer and solvent, and the specific volumes at this temperature. Values of $v_{2}$ thus obtained were in good agreement with direct pycnometric measurements on the solutions themselves.

Viscosities of the poly(trimethylene oxide) fractions in the cyclohexane were determined in a Cannon-Ubbelohde viscometer. Each fraction was studied at a minimum of four concentrations, in the range 0.03 to $2.17 \mathrm{~g} / \mathrm{dl}$, and values of the relative viscosity ranged from 1.02 to 2.03 .

\section{RESULTS AND DISCUSSION}

The binodials for the liquid-liquid phase separations were obtained by plotting values of the precipitation temperature against composition. Typical results are shown in Figure 1. The maximum of each binodial is the critical solution temperature $T_{c}$ at that particular degree of polymerization $x$. Although some of the data scatter somewhat, presumably because of the relatively low molecular weights of some of the fractions, the values of $T_{C}$ obtained are probably accurate to $\pm 0.2^{\circ} \mathrm{C}$. Their reciprocals were plotted against the molecular size function indicated by theory, in the usual manner ${ }^{7}$. The results are presented in Figure 2, where the line shown was located by least-squares analysis. The intercept gives the value of $T_{c}$ in the limit of infinite chain length, which is the $\theta$-temperature of the system. We thus obtain $\theta=27.0^{\circ} \mathrm{C}$, with an uncertainty estimated to be $\sim \pm 0.5^{\circ} \mathrm{C}$. The entropy of dilution parameter $\psi_{1}{ }^{7}$, obtained from the slope of this curve, was found to have the relatively large value 1.0 .

Values of the intrinsic viscosity $[\eta]$ of the poly(trimethylene oxide) fractions in cyclohexane at $27.0^{\circ} \mathrm{C}$ were obtained from the solution viscosities in the usual manner ${ }^{7}$; the results are given in Table 1. They are plotted logarithmically against the number-average molecular weight in Figure 3, and were found by least-squares analysis to be represented by the relationship:

$$
[\eta]=8.89 \times 10^{-4} M_{n}^{0.519}
$$

The fact that the exponent on the molecular weight is onehalf $^{7}$ within experimental error confirms cyclohexane to be a $\theta$-solvent for poly (trimethylene oxide) at $27.0^{\circ} \mathrm{C}$. Values of the unperturbed dimensions $\left\langle r^{2}\right\rangle_{0}$ are therefore directly calculable from the equation:

$$
[\eta]=\Phi\left(\left\langle r^{2}\right\rangle_{0} / M_{n}\right)^{3 / 2} M_{n}^{1 / 2}
$$

which is applicable to any random-coil polymer in a $\theta$ solvent ${ }^{7}$; under these conditions, the constant $\Phi$ has the value $2.5 \times 10^{21} \mathrm{dl} / \mathrm{mol} \mathrm{cm}^{3} 7,16$. The unperturbed dimensions thus obtained were expressed relative to $n l^{2}$, with $n=4\left(M_{n} / M_{0}\right)$, where $M_{0}=58.08$ is the molecular weight of the repeat unit, and $l^{2}=2.19 \AA^{21}$. The resulting values of the characteristic ratio are given in Table 1 and are seen to be independent of molecular weight, as expected ${ }^{4,7}$.

The average value $\left\langle r^{2}\right\rangle_{0} / n l^{2}=3.86 \pm 0.08$ is in excellent agreement with the value $3.94 \pm 0.17$ obtained from viscometric and osmometric measurements in a thermodynamically good solvent, benzene at $30^{\circ} \mathrm{C}^{1}$. The agreement may be somewhat fortuitous, however, in that it is frequently

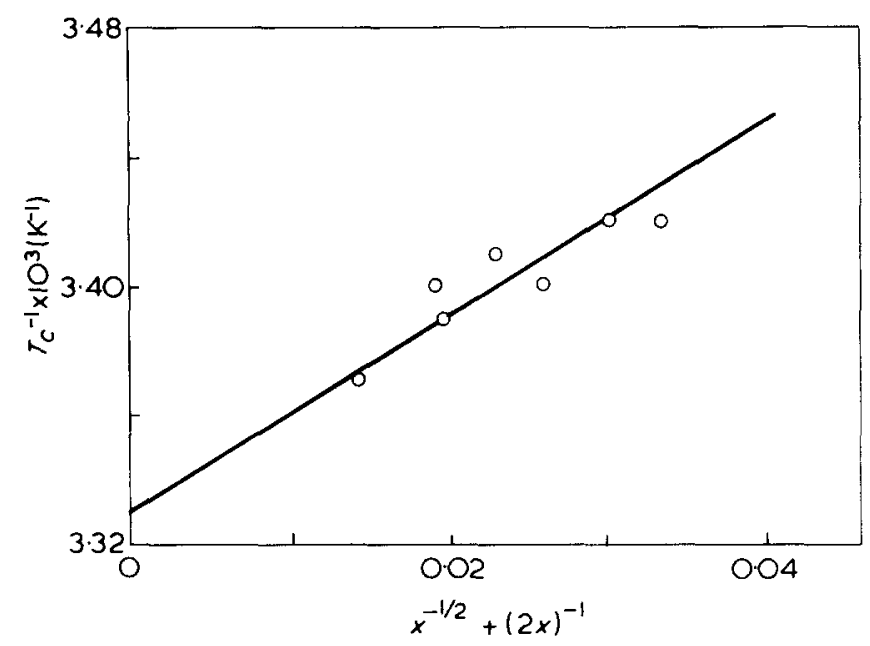

Figure 2 Extrapolation of reciprocal critical solution temperatures to obtain the $\theta$-temperature for the poly (trimethylene oxide)cyclohexane system.

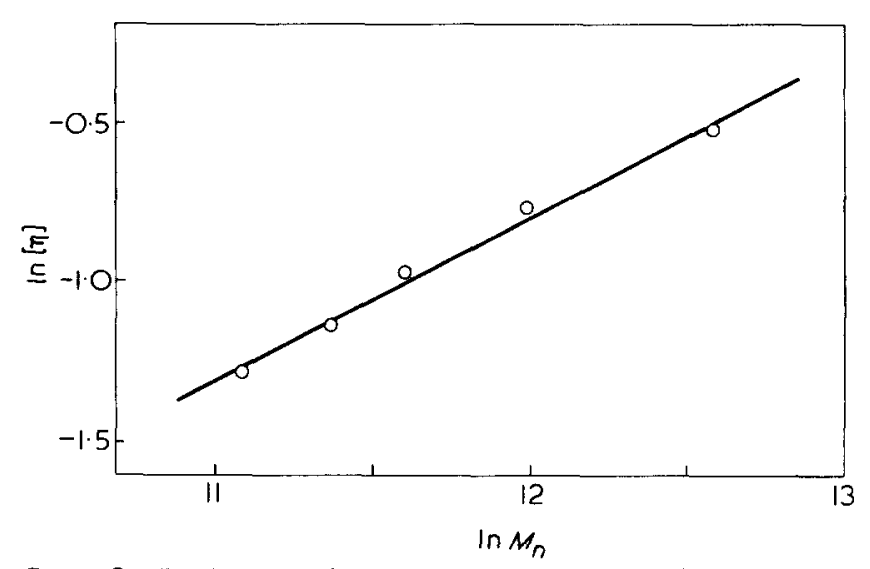

Figure 3 The intrinsic viscosity-molecular weight relationship for poly (trimethylene oxide) in cyclohexane at the $\theta$-temperature, $27.0^{\circ} \mathrm{C}$ 
Tab/e 2 Some characteristics of polyethylene, polyoxymethylene, and their alternating copolymer poly(trimethylene oxide)

\begin{tabular}{|c|c|c|c|}
\hline & PE & POM & $\mathrm{POM}_{3}$ \\
\hline $\begin{array}{l}\text { Repeat unit } \\
\left.\left\langle r^{2}\right\rangle_{0} / n\right\rangle^{2} \\
10^{3} \mathrm{~d}\left(\ln \left\langle r^{2}\right\rangle_{0}\right) / \mathrm{d} T\end{array}$ & $\begin{array}{c}-\mathrm{CH}_{2}-\mathrm{CH}_{2}- \\
7.6^{\mathrm{a}, \mathrm{b}} \\
-1.1 \mathrm{a}\end{array}$ & $\begin{array}{c}-\mathrm{CH}_{2}-\mathrm{O}- \\
12 \mathrm{c} \\
-6 \mathrm{~d}\end{array}$ & $\begin{array}{c}-\mathrm{CH}_{2}-\mathrm{CH}_{2}-\mathrm{CH}_{2}-\mathrm{O}- \\
3.9^{\mathrm{e}} \\
0.1^{\mathrm{f}}\end{array}$ \\
\hline
\end{tabular}

aThe pertinent experimental studies are cited in ref 4 . bThe actual experimental result is 6.7 at $140^{\circ} \mathrm{C}$; it was corrected to the vicinity of $25^{\circ} \mathrm{C}$ by means of the coefficient $\mathrm{d}\left(\ln \left\langle r^{2}\right\rangle_{0}\right) / \mathrm{d} T$. CApproximate value estimated from intrinsic viscosities ${ }^{13}$ obtained in a thermodynamically good solvent at $25^{\circ} \mathrm{C}$, using a value of $2.1 \times 10^{21}$ for the hydrodynamic constant $\Phi^{1.16}$. dVery approximate value estimated from intrinsic viscosities obtained in two thermodynamically good solvents at $25^{\circ}$ and $90^{\circ} \mathrm{C}$, respectively 13,14 . ePresent study. fRef 1

very difficult to correct satisfactorily for the excluded volume effects present in thermodynamically good solvents ${ }^{17,18}$. In any case, the characteristic ratio found for poly(trimethylene oxide) is unusually small, and is probably the smallest of those $e^{2,4}$ which are reliably known, to a high degree of accuracy $\dagger$. This compactness of the poly(trimethylene oxide) chain is due to its high conformational randomness; two of the skeletal bonds of the repeat unit have a preference for gauche rotational states, whereas the other two have a preference for the alternative trans states $^{1,3,5}$.

In addition, it is of instructional value to note that poly(trimethylene oxide) may be considered an alternating copolymer of ethylene and oxymethylene units, and it is thus of interest to compare its configuration-dependent properties with those of its parent homopolymers polyethylene $\left(\mathrm{CH}_{2}-\mathrm{CH}_{2}\right)_{x}$ and polyoxymethylene $\left(\mathrm{CH}_{2}-\mathrm{O}\right)_{x}$. Pertinent results are given in Table 2. Polyethylene is seen to have a relatively high value, $7.6^{1,4}$, for the characteristic ratio in the vicinity of $25^{\circ} \mathrm{C}$; polyoxymethylene, at the same temperature, has one of the highest values ${ }^{1,13}, 12 \ddagger$, reported to date. This is due to the fact that these two homopolymeric chains exhibit a preference for conformations of high spatial extension; in polyethylene the preference is for trans or planar zig-zag conformations ${ }^{4}$, and in polyoxymethylene it is for gauche states giving rise to helical sequences ${ }^{4}$. High spatial extension is not preserved, however, upon mixing the two types of units, in alternating sequence, to give poly(trimethylene oxide). The characteristic ratio of poly(trimethylene oxide) is in fact very much smaller than the corresponding value for either homopolymer, since gauche states cause disruption of the planar trans sequences, while trans states likewise disrupt the helical gauche sequences. Similarly, the large negative values reported for the temperature coefficient $\mathrm{d}\left(\ln \left\langle r^{2}\right\rangle_{0}\right) / \mathrm{d} T$ of the unperturbed dimensions of polyethylene and poly. oxymethylene are of course a direct result of the fact that the spatially extended conformations in these chains are of relatively low energy ${ }^{4}$. Again, destruction of the conformational regularity in polyethylene and in polyoxymethylene by the intermixing of these two repeat units in poly(trimethylene oxide) has the marked effect of reducing its value of $\mathrm{d}\left(\ln \left\langle r^{2}\right\rangle_{0}\right) / \mathrm{d} T$ essentially to zero'. These compari-

\footnotetext{
$\dagger$ It is of course important to use the same conventions in calculating values of the characteristic ratio in comparisons of this type. In polypeptides, for example, 'virtual bonds' of length $3.80 \AA$ are frequently used to replace three consecutive skeletal bonds, of lengths $1.53,1.32$, and $1.47 \mathrm{~A}$, respectively 4,8 . For uniformity, therefore, characteristic ratios thus calculated would have to be multiplied by the factor $\left[3.80^{2} /\left(1.53^{2}+1.32^{2}+1.47^{2}\right)\right]=2.31$.

$\ddagger$ Stockmayer and Chan ${ }^{13}$ calculate the somewhat lower value $10.5 \pm 1.5$, using $\Phi=2.5 \times 10^{21}$ rather than the value $2.1 \times 10^{21}$ we used to obtain $\left\langle r^{2}\right\rangle_{0} / n l^{2} \cong 12^{1}$. We consider the uncertainty associated with this assignment to be minor compared with other uncertainties 19,20 inherent in the use of such extrapolation methods 21 in the interpretation of viscometric data.
}

sons strikingly illustrate the very large error which may be introduced by use of the assumption ${ }^{9}$ that a chemical copolymer should have statistical properties intermediate to those of its parent homopolymers.

\section{ACKNOWLEDGEMENTS}

The authors are pleased to acknowledge financial support from the Ministry of Education of Japan, the National Science Foundation, and the Macromolecular Research Center of the University of Michigan.

\section{REFERENCES}

1 Takahashi, Y. and Mark, J. E. J. Am. Chem. Soc. 1976, 98, 000

2 'Polymer Handbook', (Eds. J. Brandrup and E. H. Immergut), 2nd Edn, Interscience, New York, 1975

3 Mark, J. E. J. Polym. Sci. (B) 1966, 4, 825

4 Flory, P. J. 'Statistical Mechanics of Chain Molecules', Interscience, New York, 1969

5 Abe, A. and Mark, J. E. J. Am. Chem. Soc. in press

6 Mark, J. E. Acc. Chem. Res. 1974, 7, 218

7 Flory, P. J. 'Principles of Polymer Chemistry', Cornell University Press, Ithaca, 1953

8 Brant, D. A., Miller, W. G. and Flory, P. J. J. Mol. Biol. $1967,23,47,67$

9 Cirlin, E. H. et al. J. Macromol. Sci. (A) 1971, 5, 981; Chen, T. Y., Ricica, P. and Shen, M. ibid, 1973. 7, 889

10 Hallman, G. M. and Whittington, S. G. Macromolecules $1973,6,386$

11 Mark, J. E. J. Polym. Sci. (Polym. Phys. Edn) 1974, 12, 1207

12 Mark, J. E. in 'Characterization of Materials in Research; Ceramics and Polymers', (Eds J. J. Burke and V. Weiss), Syracuse University Press, Syracuse, 1975

13 Stockmayer, W. H. and Chan, L.-L. J. Polym. Sci. (A-2) $1966,4,437$

14 Kokle, V. and Billmeyer, F. W. Jr. J. Polym. Sci. (B) 1965, 3,47

15 Mark, J. E. and Flory, P. J. J. Am. Chem. Soc. 1965, 87, 1423

16 McIntyre, D. et al.J. Phys. Chem. 1962, 66, 1932; Berry, G. C J. Chem. Phys. 1967, 46, 1338

17 Orofino, T. A. and Flory, P. J. J. Chem. Phys. 1957, 26, 1067

18 Fujita, H. and Norisuye, T. J. Chem. Phys. 1970, 52, 1115

19 Flory, P. J. Makromol. Chem. 1966, 98, 128

20 Bluestone, S., Mark, J. E. and Flory, P. J. Macromolecules $1974,7,325$

21 Kurata, M. and Stockmayer, W. H. Adv. Polym. Sci. 1963, 3,196

\section{Note added in proof}

Also pertinent with regard to the statistical properties of chemical copolymers is the fact that the unperturbed dimensions of poly(vinylidene fluoride) $\left[\mathrm{CF}_{2}-\mathrm{CH}_{2}-\right]_{x}$ are quite different from those of polytetrafluoroethylene $\left[\mathrm{CF}_{2}-\mathrm{CF}_{2}-\right]_{x}$ and polyethylene $\left[\mathrm{CH}_{2}-\mathrm{CH}_{2}\right]_{x}$ (Tonelli, A. E. Macromolecules submitted). 